\title{
Morphological validation of a novel bi-material 3D-printed model of temporal bone for middle ear surgery education
}

\author{
Jordan Chauvelot ${ }^{1}$, Cedric Laurent ${ }^{2}$, Gaël Le Coz $^{2}$, Jean-Philippe Jehl ${ }^{3}$, Nguyen Tran ${ }^{4}$, \\ Marta Szczetynska ${ }^{2}$, Abdelhadi Moufki ${ }^{2}$, Anne-Sophie Bonnet ${ }^{2}$, Cecile Parietti-Winkler ${ }^{1}$ \\ ${ }^{1}$ ENT Department, University Hospital of Nancy, Vandœuvre-lès-Nancy, France; ${ }^{2}$ CNRS, LEM3, UMR 7239, University of Lorraine, Metz, \\ France; ${ }^{3}$ CNRS, IJL, UMR 7198, University of Lorraine, Campus Artem, Nancy, France; ${ }^{4}$ School of Surgery Nancy-Lorraine, Faculty of Medicine, \\ Vandœuvre-Lès-Nancy, France \\ Contributions: (I) Conception and design: J Chauvelot, C Laurent, G Le Coz, A Moufki, AS Bonnet, C Parietti-Winkler; (II) Administrative support: \\ JP Jehl, N Tran, AS Bonnet, C Parietti-Winkler; (III) Provision of study materials or patients: C Laurent, G Le Coz, N Tran, C Parietti-Winkler; \\ (IV) Collection and assembly of data: J Chauvelot, C Laurent, G Le Coz; (V) Data analysis and interpretation: J Chauvelot, C Laurent, G Le Coz, M \\ Szczetynska, A Moufki, AS Bonnet, C Parietti-Winkler; (VI) Manuscript writing: All authors; (VII) Final approval of manuscript: All authors. \\ Correspondence to: Jordan Chauvelot. Service ORL, CHRU de Nancy, 5 rue du Morvan, 54500 Vandoeuvre-Les-Nancy, France. \\ Email: jordanchauvelot@yahoo.fr.
}

Background: A new model of 3D-printed temporal bone with an innovative distinction between soft and hard tissues is described and presented in the present study. An original method is reported to quantify the model's ability to reproduce the complex anatomy of this region.

Methods: A CT-scan of temporal bone was segmented and prepared to obtain 3D files adapted to multimaterial printing technique. A final product was obtained with two different resins differentiating hard from soft tissues. The reliability of the anatomy was evaluated by comparing the original CT-scan and the preprocessed files sent to the printer in a first step, and by quantifying the printing technique in a second step. Firstly, we evaluated the segmentation and mesh correction steps by segmenting each anatomical region in the CT-scan by two different other operators without mesh corrections, and by computing distances between the obtained geometries and the pre-processed ones. Secondly, we evaluated the printing technique by comparing the printed geometry imaged using $\mu \mathrm{CT}$ with the pre-processed one.

Results: The evaluation of the segmentation and mesh correction steps revealed that the distance between both geometries was globally less that one millimeter for each anatomical region and close to zero for regions such as temporal bone, semicircular canals or facial nerve. The evaluation of the printing technique revealed mismatches of $0.045 \pm 0.424 \mathrm{~mm}$ for soft and $-0.093 \pm 0.240 \mathrm{~mm}$ for hard tissues between the initial prepared geometry and the actual printed model.

Conclusions: While other reported models for temporal bone are simpler and have only been validated subjectively, we objectively demonstrated in the present study that our novel artificial bi-material temporal bone is consistent with the anatomy and thus could be considered into ENT surgical education programs. The methodology used in this study is quantitative, inspired by engineer sciences, making it the first of its kind. The validity of the manufacturing process has also been verified and could, therefore, be extended to other specialties, emphasizing the importance of cross-disciplinary collaborations concerning new technologies.

Keywords: Artificial temporal bone; 3D printing; surgical training; educational tool; simulation

Submitted Jan 26, 2020. Accepted for publication Feb 24, 2020.

doi: $10.21037 /$ atm.2020.03.14

View this article at: http://dx.doi.org/10.21037/atm.2020.03.14 


\section{Introduction}

Surgery requires thorough anatomical knowledge and acquisition of technical skills that inevitably required long and repetitive hours of practice. Obviously, surgical training, or evaluation of trainees, based on supervised practice of real surgeries should not be acceptable $(1,2)$ anymore, especially in the first steps of surgical education. Middle ear surgery concerns a very small anatomical region, volume of which does not exceed 5 to 6 drops of water. Indeed, this region includes the smallest bone of the whole human body: the stapes (not exceeding $3 \mathrm{~mm}$ ) (3) and many other at-risk structures such as facial nerve, dura-mater, sigmoid sinus, balance and auditory sensors. Consequently, the main part of middle ear surgery is performed with operative microscope, during which the otologic surgeon proceeds to millimetric gestures with an extreme precision and tactfulness. Such gestures, realized without direct vision and during several hours, are tiring and represent a challenge for surgeons. In such a context, millimetric errors may have dramatic consequences, including facial paralysis (4,5), labyrinthine effraction with hearing loss and vertigo, or dura mater effraction with a cerebrospinal fluid leak (6-9). Prior to real surgeries, it seems crucial that the future surgeons can rehearse and be evaluated in the most realistic possible context, generally consisting in cadaveric human samples. However, such traditional surgical educational tools are rare, costly and even hazardous because they may constitute potential vectors of diseases $(10,11)$. Besides, unlike in other surgical specialties, there is no animal model that can be used as educational tool in middle surgery $(12,13)$.

Alternatively, simulation of surgical procedures using virtual reality-based simulators (14-17). or artificial realistic three-dimensional (3D) models (18-20) are of great interest. Such simulators are now available for middle ear surgery education thanks to recent advances in these new technologies. They may represent complementary educational tools to cadaveric samples, facilitating anatomical knowledge and technical skills acquisition in learners, without risk or stressful context neither for patients nor for learners. In the present study, a 3D-printed bi-material temporal bones (TB) has been designed for surgical training, using multi-material printing, which constitutes a well-known technique widely applied to create high-resolution polymeric biomaterials (21-24) allowing a distinction between bone and soft tissues. However, before being used in pedagogic programs, or even more for learners' certification, simulation devices must be validated as educational tools. Indeed, learning with surgical simulators makes sense only if these devices both provide learning context and present characteristics close enough to the real clinical situation (14). Concerning artificial $\mathrm{TB}$, for example, their geometry needs to be consistent with the real TB anatomy with minimal discrepancies. Unfortunately, the realism of currently available artificial TB models, in comparison with a real TB, is debatable. Indeed, their validation as reliable educational tools is rare in literature and when exists, it is most of the time based on subjective qualitative comparisons (18-20,25). Subjective evaluation through dissection performed by expert appears limited, and there is a lack of further objective validation and quantitative methodologies. Such validation would allow concluding to the clinical suitability of the simulation device. To fill this gap, one way of assessing the geometry of such a simulation device could be to compare the artificial model with the source patient's real anatomy. Such an objective methodology is inspired from the field of engineering sciences (26) and allows identification of 3 potential sources of errors, leading to mismatches between the real patient's anatomy (related to the source patient's TB CT-scan) and the artificial TB. The first one may occur during the segmentation step performed manually by clinicians from the Digital Imaging and Communications in Medicine (DICOM) files that inevitably involved a subjective interpretation of the different structure limits. The second one may result from the smoothing and simplifications of the mesh patterns after the segmentation step [required to prepare the files for 3D-printing and result in appropriated STereoLithography (STL) files] $(27,28)$, potentially leading to discrepancies with the initial anatomy of DICOM files. The last potential source of error may come from the multi-material 3D-printing process that can lead to mismatches between the STL files used for printing and the actual printed device.

As a result, the aim of the present study was to assess the ability of our new 3D-printed bi-material artificial TB to reproduce the complex anatomy of the source $\mathrm{TB}$, proving, all at once, the reliability of its manufacturing process.

\section{Methods}

An anonymous adult female, without history of middle ear pathology, underwent a TB CT-scan with a standard protocol for TB examination achieved by a "VCT lightspeed-General Electric healthcare Chicago Illinois U.S” with $0.625 \mathrm{~mm}$ sections. Both an otologist expert and 


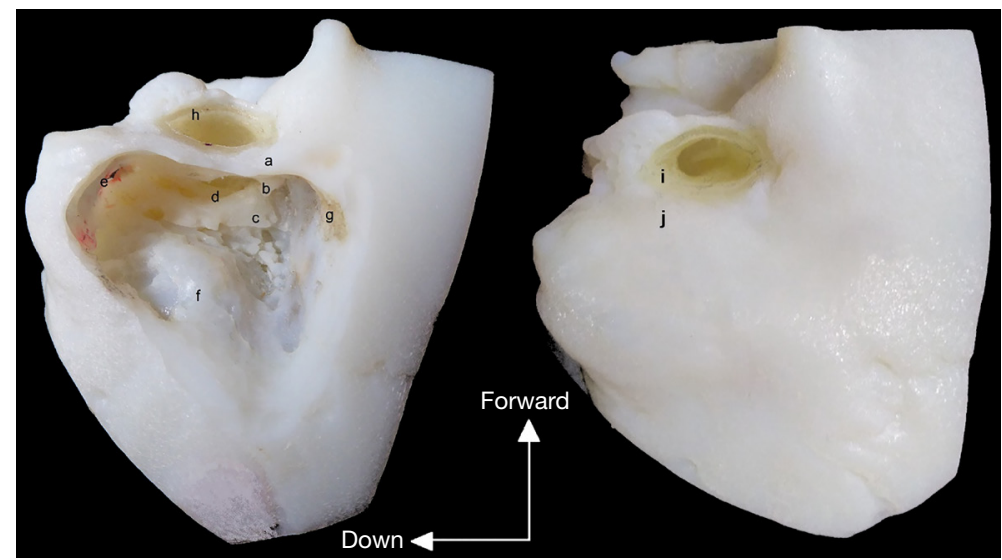

Figure 1 The bi-material 3D-printed temporal bone in operating position. Left: dissected model with identification of both hard: a: cortical bone; b: short process of the incus; c: external semi-circular canal) and soft (d: facial nerve; e: digastric crest; f: sigmoid sinus; g: dura mater; h: external auditory canal) resins. Right: full model with visualization of both soft (i: external auditory duct) and hard (j: mastoid bone) resins.

a neuroradiologist expert corroborated that the whole TB was normal (29), namely: a good airing of mastoid cells, a good shape and position of the ear-drum, ossicles, facial nerve and inner-ear components and finally, no protrusion of sigmoid sinus. The left side has been chosen arbitrarily. The DICOM files obtained were then segmented (by otologists and medical engineers with Blender (30) free software) in order to extract: outer ear, ear-drum, middle ear with malleus, incus and stapes, inner ear with the whole labyrinth (cochlea, vestibule with semi-circular canals, fenestra vestibuli and fenestra cochleae), dura mater, sigmoid sinus and the facial nerve (with chorda tympani). A mesh model under a standard tessellation language (STL) format was then obtained and smoothed to fit for 3D-printing. We used different hardness of resins ("shores") for hard (bone) and soft (facial nerve, chorda tympani, dura mater, sigmoid sinus, ear drum, fenestra vestibuli, fenestra cochleae, membranous semi-circular canals and skin) tissues. The whole and dissected final printed models are shown on Figure 1.

\section{Evaluation of the CT-scan segmentation and mesh correction steps}

The final device has been compared to the initial CT-scan of the source patient, considered by experts as "normal" concerning ear structures and settled as a basis for the design of this device.

The DICOM files of the TB CT-scan model were segmented manually and individually by two different operators (to maximize segmentation variations), slice by slice (Figure 2), with "3D-slicer" software (31). The different anatomical structures were extracted without any further smoothing process (to emphasize the impact of mesh corrections) and the two sets of patterns thus obtained were compared to the STL files used for 3D-printing.

Point-to-surface distances (shortest node distances between two frames, Figure 3) were then calculated using algorithms based on the vtk visualization toolkit (32). The comparison between both geometries of the STL files used for $3 \mathrm{D}$ printing, yet smoothed, and the new ones, devoid of any smooth correction, requires the same reference frame. Thus, a preliminary manual repositioning was performed for each anatomical region [bone, vein (sigmoid sinus), semicircular canals, facial nerve, malleus, incus, stapes, fenestra cochleae and external auditory canal (with eardrum)], using bone structures of the new segmentation as a reference to compute the suited transformation. This procedure permitted to evaluate both the shape and the position of each structure in the whole model.

\section{Evaluation of the multi-material 3D printing technique}

This second characterization aimed at evaluating the mismatches related to the bi-material 3D printing process, by comparison between both geometries of the initial STL files and the actual 3D-printed geometry. High-resolution micro-Computed Tomography $(\mu \mathrm{CT})$ scan was required to distinguish both resins but generated heavy files. Only a fragment of the whole TB was then selected, enabling 

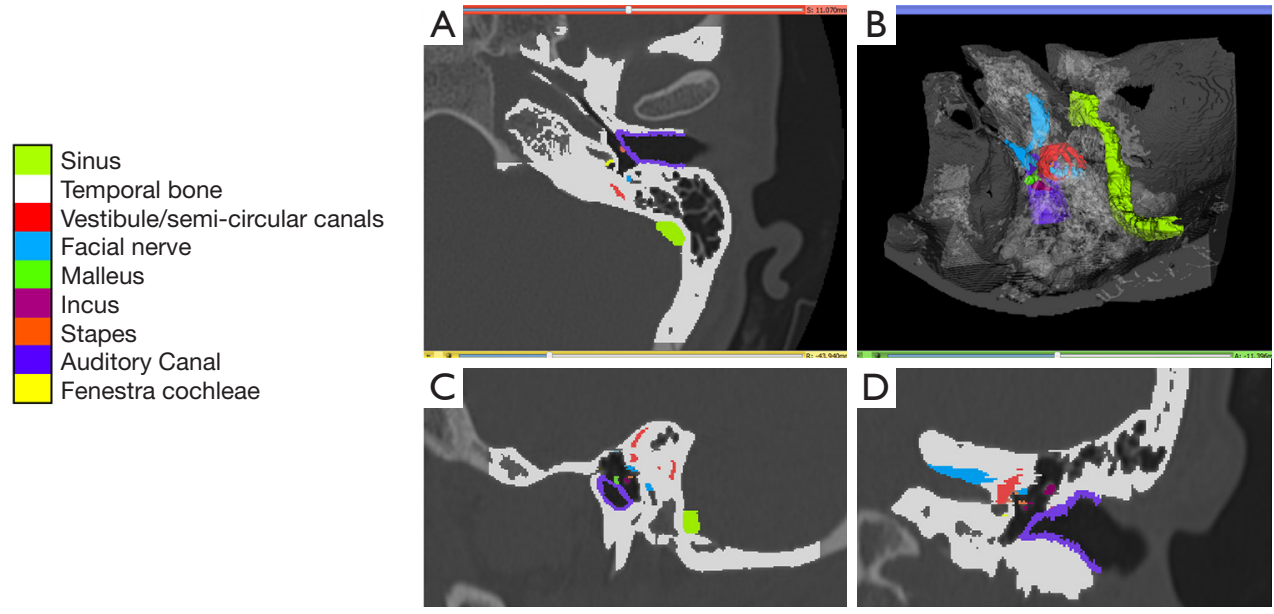

Figure 2 Manual segmentation of anatomical structures within the CT-scan. (A) axial view; (B) global 3D view; (C) sagittal view; (D) frontal view.

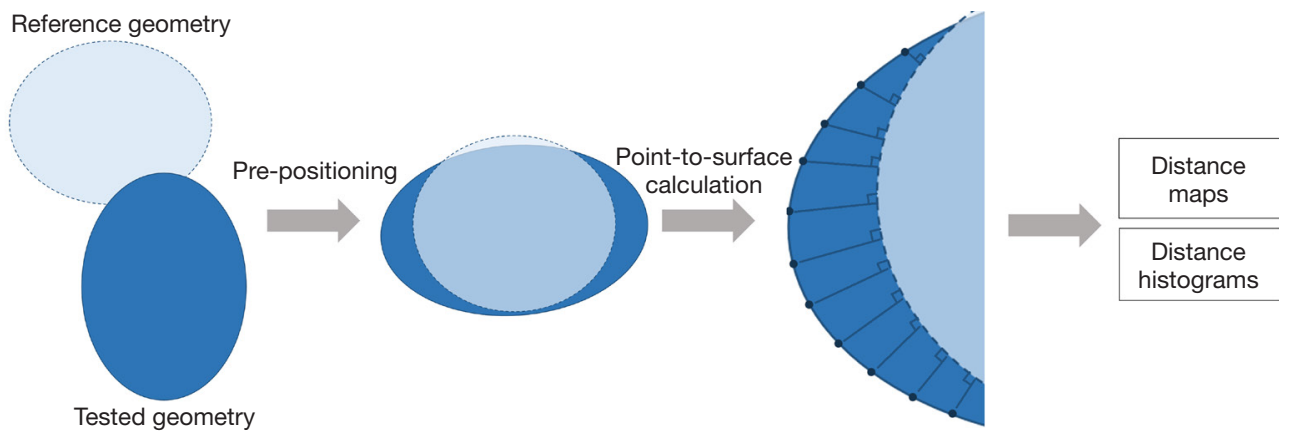

Figure 3 Explanatory diagram of the point-to-surface distances calculation. "Reference geometry" represents the segmentation of the CTscan devoid of correction. "Tested geometry" corresponds to the geometry with mesh corrections.

images with a sufficient resolution and contrast to perform the multi-material comparison between both geometries (Figure 4). The $\mu \mathrm{CT}$ was performed using a Nanotom Phoenix device (General Electrics) with a voxel size of $10.4 \times 10.4 \times 10.4 \mu^{3}$ and a window of $23.8 \times 23.8 \times 23.8 \mathrm{~mm}^{3}$. The materials constituting soft and hard tissues were then segmented manually from the $\mu \mathrm{CT}$ images using the 3D-slicer (31) and the geometries were finally extracted without any smoothing process to keep a maximum of information (Figure 4C).

This geometry was compared to the initial STL files used for 3D-printing, as described previously (pointto-surface distances calculation and distance maps). To achieve this, the fragment imaged in $\mu \mathrm{CT}$ was extracted from the STL files and positioned in the same frame that the reconstructed printed geometry. The point-to-surface distance was then computed from every point of the STL files, using the $\mu \mathrm{CT}$-based geometry as a reference.

\section{Results}

\section{Evaluation of the segmentation and mesh correction steps}

The comparison between the STL files and the geometry of each anatomical region issued from a separate segmentation of the DICOM files, without any mesh correction, was performed. An example of such comparison is given in Figure 5 for bone tissue. Geometries issued from the manual segmentation without any mesh preparation (Figure 5A) and the STL mesh files corrected and prepared for 3D printing (Figure $5 B$ ) were compared by computing the point-to-surface distances map using the new rough segmentation files as a reference (Figure $5 D$ ). This approach 


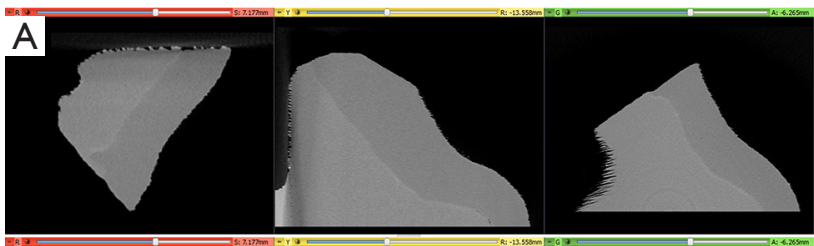

\section{C}
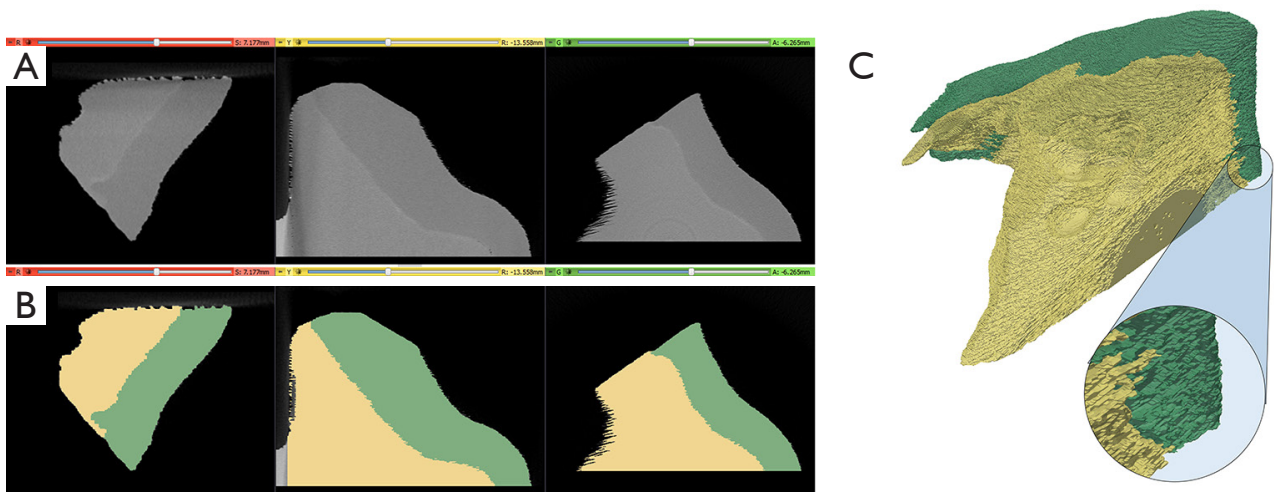

Figure $4 \mu \mathrm{CT}$ acquisition (A) and manual segmentation (B) of a fragment of the printed TB model. $\mu \mathrm{CT}$ data are used to generate a $3 \mathrm{D}$ model (C) to be compared with the initial STL files sent to the 3D printer.

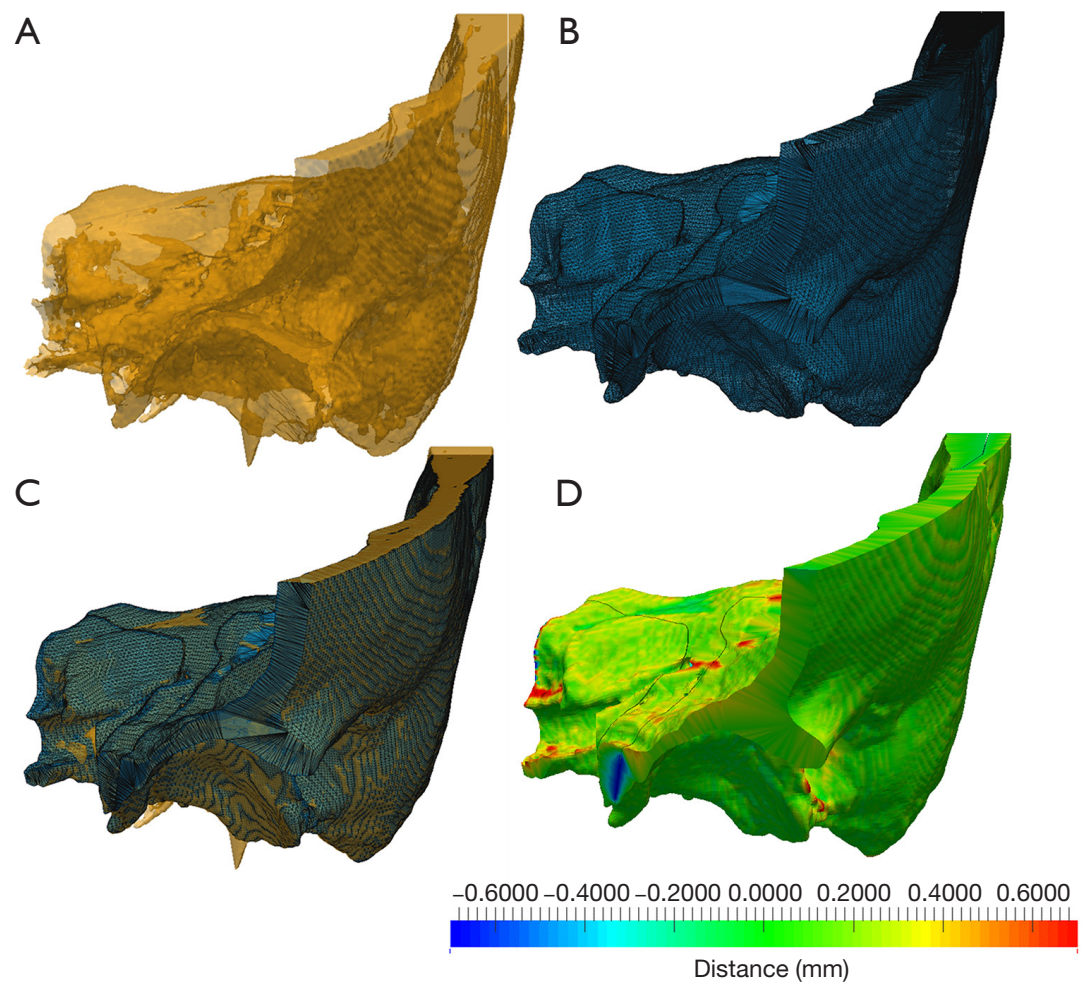

Figure 5 Example of comparison between rough reconstructed geometry issued from manual segmentation of the DICOM files (A) and the STL mesh files corrected and prepared for 3D printing (B). A superposition of both geometries is given in (C), and a distance map between these two geometries is calculated (D).

was applied by two different operators to each anatomical region present in the $\mathrm{TB}$ model, and the resulting distance maps are summarized in Figures 5,6,7. Figure 5 provides a qualitative overview of the superposition of frames, with a majority of green color, equivalent to zero distance between geometries. Blue colored (extreme negative distances) and red colored (extreme positive distances) surfaces are rare and scattered. Figures 6,7 record quantitative results (each mesh node distance is calculated) with best results for "sinus" (Figure $6 B, 0.02 \pm 0.37 \mathrm{~mm}$ for operator \#2), 
A

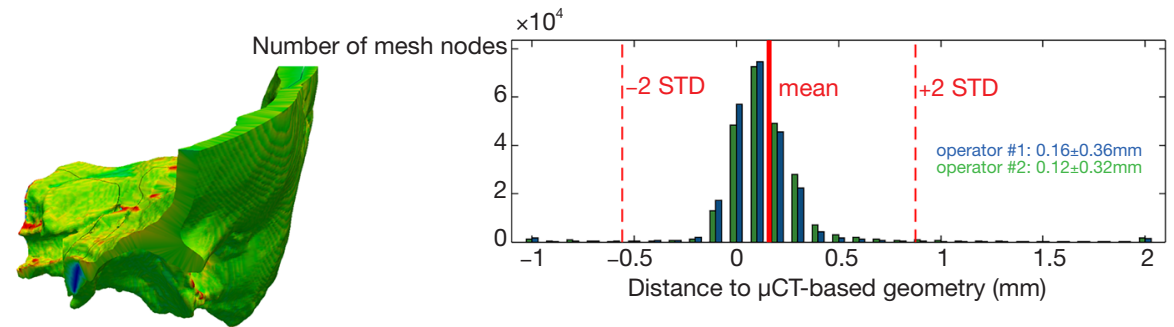

B

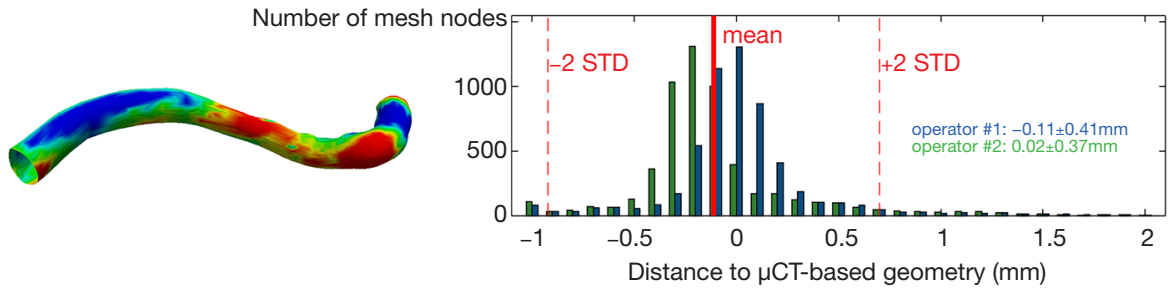

C
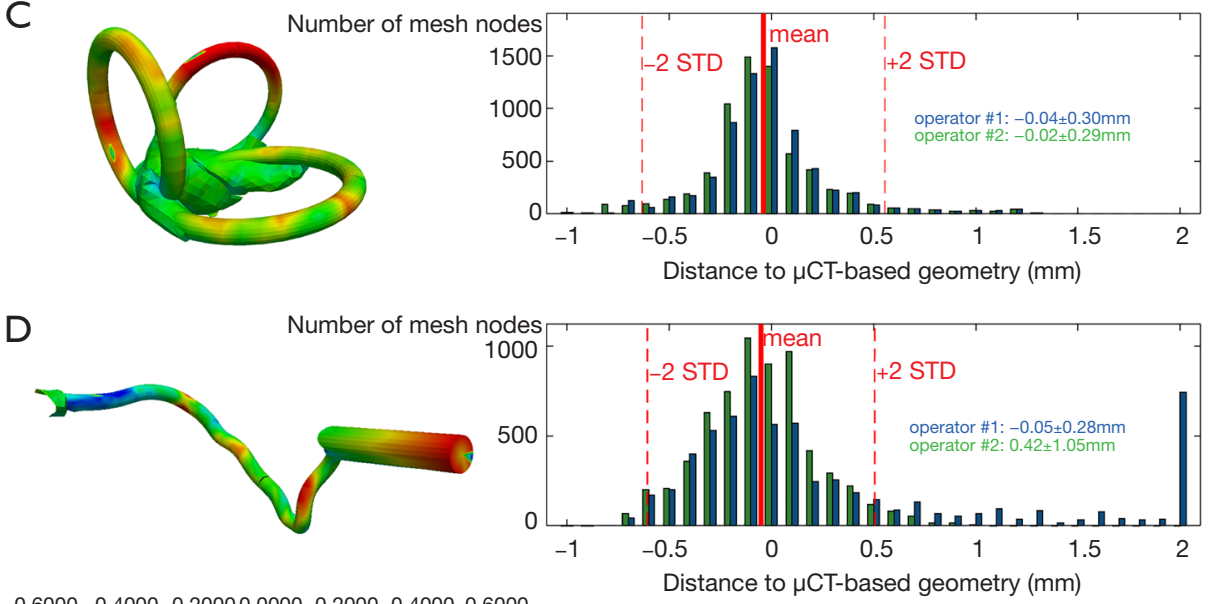

$-0.6000-0.4000-0.20000 .0000 \quad 0.2000 \quad 0.4000 \quad 0.6000$ 1

Distance $(\mathrm{mm})$

Figure 6 Distances between the rough reconstructed geometry issued from manual segmentation of the DICOM files and the STL mesh files corrected and prepared for 3D printing, for temporal bone (A), sinus (B), vestibule/semicircular canals (C) and facial nerve (D).

"vestibule/semicircular canals" (Figure 6C, $-0.02 \pm 0.29 \mathrm{~mm}$ for operator \#2 and $-0.04 \pm 0.30 \mathrm{~mm}$ for operator \#1) and "facial nerve" (Figure $6 D,-0.05 \pm 0.28 \mathrm{~mm}$ for operator \#1). As observed in Figure 7, maximal point-to-surface distances were found for the middle ear with errors around $1 \mathrm{~mm}$ for malleus (Figure $7 A, 1.01 \pm 0.66 \mathrm{~mm}$ for both operators) and fenestra cochleae (Figure $7 E, 1.12 \pm 0.40 \mathrm{~mm}$ for both operators) with globally small ranges (around millimeter) except one and only: the distal extremity of the auditory canal (Figure $7 D)$ which differs higher than $4 \mathrm{~mm}(0.66 \pm 1.25$ $\mathrm{mm}$ for operator \#1 and $0.67 \pm 1.25 \mathrm{~mm}$ for operator \#2). Globally, the reconstructed geometries agree with the STL files prepared for $3 \mathrm{D}$ printing within a mean error of less than $1 \mathrm{~mm}$.

\section{Evaluation of the $3 D$ printing technique}

With the same process, qualitative (like in Figure 5) and quantitative (like in Figures 6,7) comparisons were performed between the geometry of STL files used for $3 \mathrm{D}$-printing process and the actual printed $\mathrm{TB}$ model (reconstructions issued from $\mu \mathrm{CT}$ ). Qualitative results are represented in Figure 8. We observed that the distance between both geometries was generally below $0.5 \mathrm{~mm}$ except for precise regions that were not easily identified from manual segmentation.

A quantitative analysis (Figure 9) indicated that the mean distances between $\mu \mathrm{CT}$-reconstructed geometry and STL initial files were respectively $0.045 \pm 0.424 \mathrm{~mm}$ and 
A

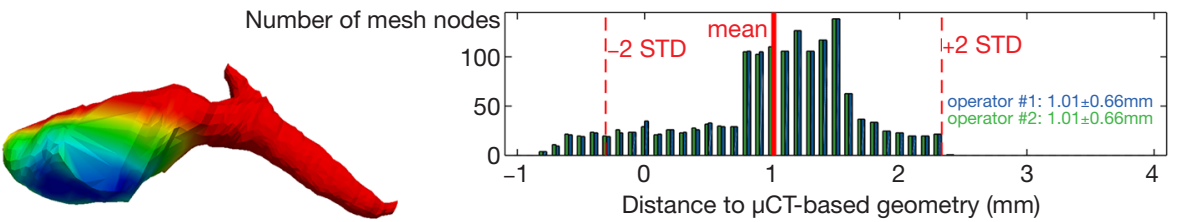

B

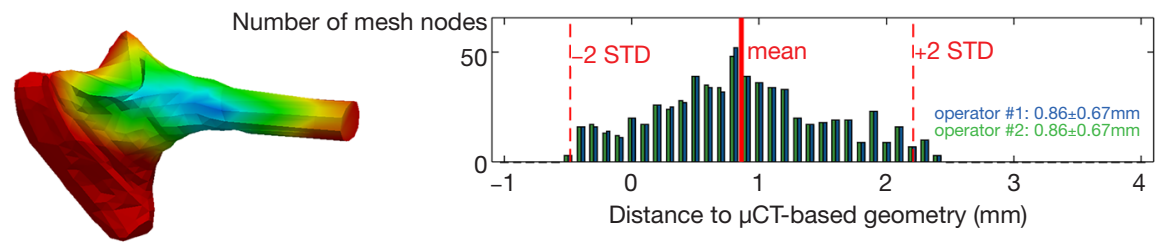

C

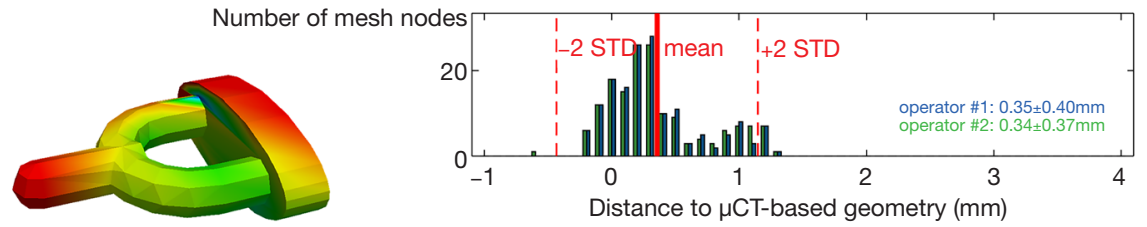

D

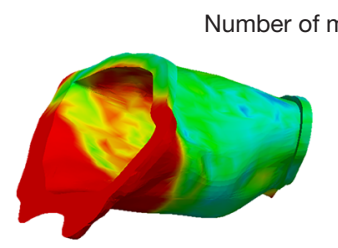

Distance to $\mu$ CT-based geometry $(\mathrm{mm})$

$\mathrm{E}$

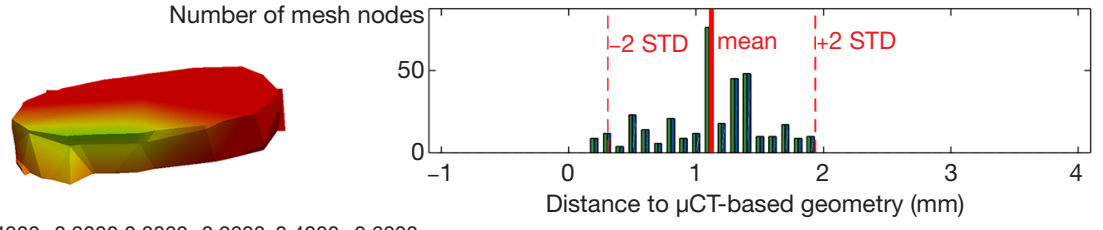

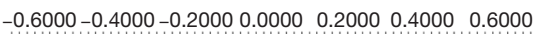

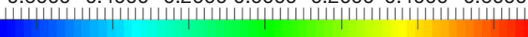

Distance (mm)

Figure 7 Distances between the rough reconstructed geometry issued from manual segmentation of the DICOM files and the STL mesh files corrected and prepared for 3D printing, for malleus (A), incus (B), stapes (C), auditory canal (D) and fenestra cochleae (E).

$-0.093 \pm 0.240 \mathrm{~mm}$ for soft and hard tissues.

\section{Discussion}

In the current contribution, we characterized the anatomical reliability of an innovative bi-material model of TB, created by $3 \mathrm{D}$-printing technique, by comparing morphology of the final object to the pre-processed files and the initial CTscan. Sources of errors have been identified and quantified. Thereafter, the place of this tool in surgical simulation and training is discussed.

Recent reforms guided by the Accreditation Council for Graduate Medical Education (ACGME), with restrictions on duty hours for instance, supposed to reduce medical errors on one hand, may have, on the other hand, a negative impact on the surgical confidence and competence of residents (33-35). This could be offset by improved surgical simulators, moreover encouraged by institutions (1).

So far, cadaver dissection constitutes the training "gold standard", but is associated with different limitations. Corpses may carry infectious agents such as Mycobacterium tuberculosis, Hepatitis B or C viruses, and prions associated with encephalopathies such as Creutzfeldt-Jakob disease $(11,36)$. Exposure to formaldehyde, when used for fixation, may potentially be carcinogen (37). Moreover, cost and availability widely vary between countries, or between regions in a same country, and may be prohibitive in case of supply limitations due to financial or cultural issues. Referring to 
A

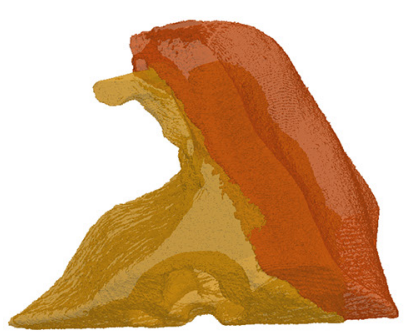

B

C
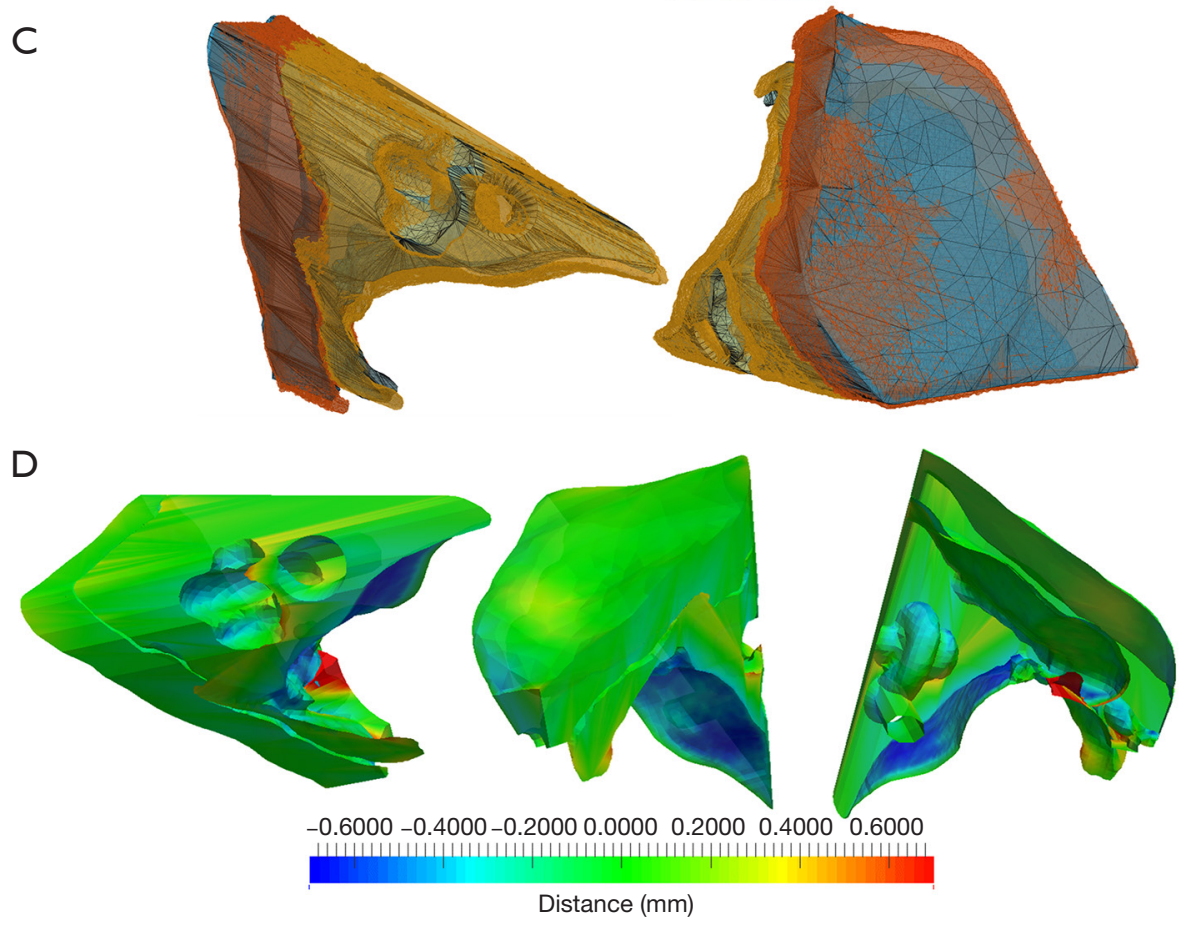

Figure 8 Qualitative comparison between STL files sent to the 3D printer and the actual geometry of the printed model issued from $\mu C T$. (A) $\mu \mathrm{CT}$ reconstruction of soft and hard tissues from the bi-material printed model. (B) Corrected STL files initially sent to the 3D printer, separated into soft and hard tissues. (C,D) Superposition of the geometries before and after printing.
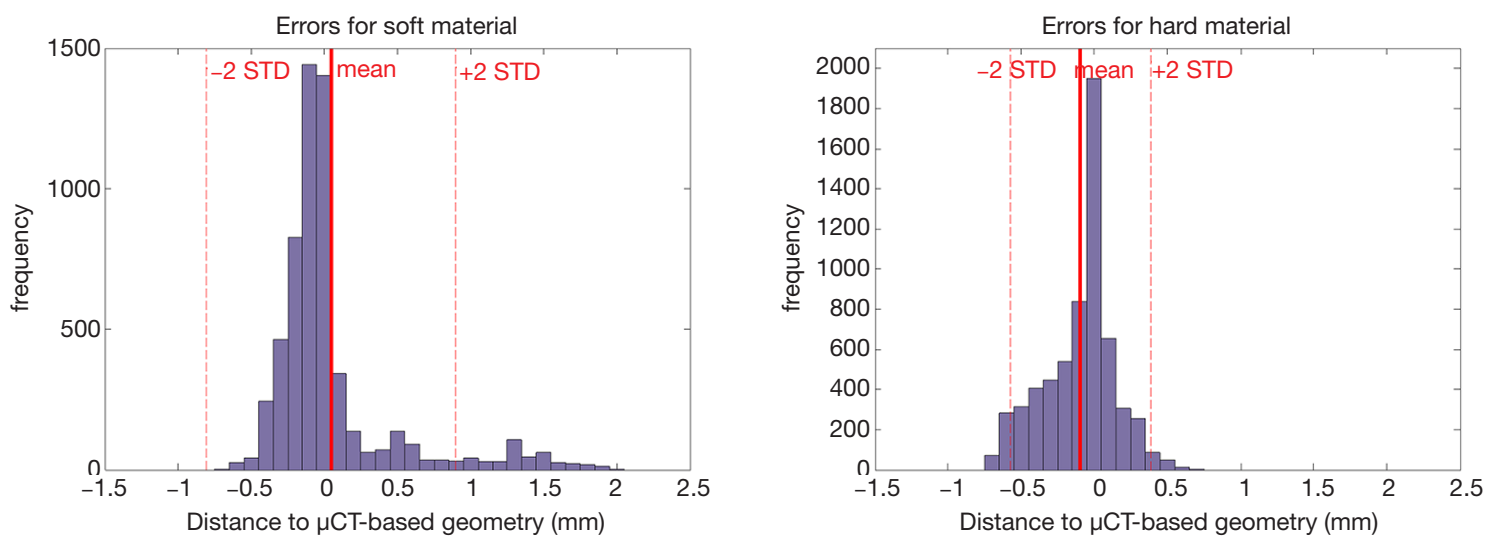

Figure 9 Quantitative comparison between STL files geometry sent to the printing machine, and the actual TB model issued from $\mu$ CT reconstructions for both soft and hard materials. 
a study from Pakistan in 2011, 37\% of medical students avoided cadaveric dissection due to moral or ethical grounds and another $18.6 \%$ for religious reasons (38). An additional downside in using cadaver TB for training is the inability to either standardize specimens for training and testing skills or provide customized scenarios for teaching. This is made possible somewhat with virtual reality or synthetic TB simulators (14). Trainees can learn through trial and error approach and repeat infinitively surgical tasks both to learn anatomy and relationships between $3 \mathrm{D}$ structures and to improve knowledge and technical skills (gesture precision, speed etc.) (39). It is worthy to emphasize that simulators do not replace traditional educational tools (such as training on cadaveric TB or supervised practice of real surgeries in the last learning steps), but they appear complementary, allowing to widen the range of educational tools, particularly for the very first steps of training. Then, these educational simulators [virtual reality like Voxelman (14), or artificial tools like our 3D-printed TB], combined with traditional educational tool within an education program, allow a step by step tailored education.

In recent years, 3D printing technology benefited from major improvements while the generated costs dropped significantly, allowing extending its applications to the field of medical use. Multi-material printing technology used in the present study resulted in a synthetic final model reproducing both soft and hard tissues, in different colors and textures, when most of reported models settle for hard tissues only $(18,20,40,41)$.

By 2007, Suzuki et al. (42). developed models with polyamide nylon and glass beads, primarily used for teaching anatomy, even if the authors discussed their potential as a cadaver specimen substitution in TB surgical training. In 2010, Bakhos et al. (18) used white resin to reproduce the bony anatomy of the TB based on CT scans of cadaveric specimens. In 2013, Mick et al. (43) developed TB prototypes with an improved similarity to human, with multiple colors, using plaster powder and a binding agent containing cyanoacrylate. The middle fossa plate was coated with painted latex to simulate dura mater. In 2014, Hochman et al. (44) used four different binding agents for infiltration of the powder material, and solved the problem of support material filling void spaces within the model during printing. In 2015, Rose et al. (19) managed, with an additive manufacturing technique, to create a pseudo bi-material temporal bone by varying ratios of multiple thermoset polymers, achieving unique biomechanical properties for different structures, increasing anatomical realism of 3D models. However, the question of the realism of such available $3 \mathrm{D}$ models is still to be addressed to conclude to their suitability as educational tools.

Many artificial simulators are daily used in surgery schools, even if their reliability regarding surgical anatomy is neither evaluated nor confirmed. However, realism of surgical artificial simulator geometry appears to be a main parameter for the transferability of technical skills acquired by learners during training, in the real surgical practices $(45,46)$. Only a few studies in literature assess the realism of artificial TB, and rely on subjective evaluations, with questionnaires (18). To our knowledge, our study is the first in literature to attempt to develop an objective methodology of geometry validation of 3D-printing surgical simulators. For this purpose, we identified and quantified the different sources of possible errors in the manufacturing process, as usually practiced in the field of engineering sciences $(47,48)$.

First, prior to $3 \mathrm{D}$-printing, segmented regions from the DICOM files of TB CT-scan require mesh corrections in order to smooth geometries and delete the artifacts that may result from the segmentation step. These mandatory corrections consist in minor adjustments but could become significant when concerning very small regions such as middle ear. They should then be taken into consideration and even evaluated, like in the present study. Moreover, errors due to misinterpretation of the anatomical regions are inevitable. The comparison between both geometries displays only very slight mismatches, inferior to $1 \mathrm{~mm}$, for the components of middle ear. While these results cannot be compared with those of other studies since this is the first study of its kind, they may still be discussed. The higher mismatch (up to $4 \mathrm{~mm}$ ) was observed for skin of the lateral side of the auditory canal which corresponds to the cartilaginous duct and therefore does not have any consequence since only the bony duct is considered in our TB model. We can then consider it as an artifact. This may be corrected in future versions of the proposed model but has been voluntarily retained in the current study in order to emphasize such inherent risks due to manual segmentation. Either way, we have to notice that this only structure, not of primary importance, is below our expectations in relation to the whole object which displays a mean mismatch globally inferior to $1 \mathrm{~mm}$ and most of the time inferior to $0.43 \mathrm{~mm}$ : "temporal bone" (Figure 6A, $0.15 \pm 0.35 \mathrm{~mm}$ for operator $\# 1$ and $0.12 \pm 0.32 \mathrm{~mm}$ for operator \#2), "sinus" (Figure $6 B$, $-0.11 \pm 0.40 \mathrm{~mm}$ for operator \#1 and $0.02 \pm 0.37 \mathrm{~mm}$ for operator \#2), "vestibule/semicircular canals" (Figure 6C, $-0.04 \pm 0.30 \mathrm{~mm}$ for operator \#1 and $-0.02 \pm 0.29 \mathrm{~mm}$ for 
operator \#2), "facial nerve" (Figure 6D, $-0.05 \pm 0.28 \mathrm{~mm}$ for operator \#1 and $0.42 \pm 1.05 \mathrm{~mm}$ for operator \#2) and "stapes" (Figure $7 C, 0.35 \pm 0.39 \mathrm{~mm}$ for operator \#1 and $0.34 \pm 0.37$ for operator \#2). It is worthy to note that separate segmentations performed by two different operators did not result in major differences, indicating that the segmentation does not constitute the major source of reported errors.

Secondly, the accuracy of the printing technique was assessed by comparing the geometry of the STL files (to be printed) to the obtained printed geometry imaged using $\mu \mathrm{CT}$. This was limited to a fragment of the whole TB model because of the power needed to differentiate both resins with $\mathrm{X}$-ray signals on high-resolution geometries, generating heavy files. Usable files of the whole object did not allow to differentiate materials satisfactorily and comparisons would have been uncertain. As a result, a comparative study was performed between these two partial geometries, and concluded to differences lower than $100 \mu \mathrm{m}$ for both soft and hard tissues. These differences may be attributed to the errors inherent to manual segmentation of the CT images (DICOM of the printed TB), and the resolution of the $3 \mathrm{D}$ printing machine; this would imply that the real error, generated by the printing process, is nearly inexistent.

Overall, the morphological analysis concluded to a high correspondence between the initial source patient's TB CT scan, the corrected STL files prepared for 3D printing and the resulting $3 \mathrm{D}$ artificial $\mathrm{TB}$. It may be considered that such errors, globally inferior to $1 \mathrm{~mm}$, are not of primary importance compared to the inherent $\mathrm{TB}$ anatomy variability in normal subjects (49-51). Indeed, Quam et al. (52) showed, in a series of cadaver dissections, that even a very small part such as the short process length of the incus for instance,

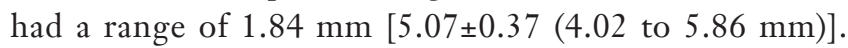
Kamrava et al. (3) showed that variations of stapes height are above $2 \mathrm{~mm}$ and range from 2 to $5.5 \mathrm{~mm}$. Singh et al. (50) showed that the variability of chorda tympani's position in the mastoid can reach more than 10 millimeters (distance of the chorda tympani from the facial nerve posterior genu was $11.9 \pm 3.3 \mathrm{~mm})$ without any statistical difference $(\mathrm{P}=0.08)$ between subjects. Beyond inter-human variability in size, shape or position of structures within the $\mathrm{TB}$, we could consider intra-observer and inter-observer variations of interpretation: Frangi et al. (53) showed in a 3D cardiac segmentation study that intra-observer variability reach 0.556 $\mathrm{mm}$ for manual and $1.785 \mathrm{~mm}$ for automatic landmarking while inter-observer variability reach $0.908 \mathrm{~mm}$ for manual and even $2.003 \mathrm{~mm}$ for automatic landmarking. In matter of otology, Iyaniwura et al. (51) observed an absolute interobserver variability average difference of $0.90 \pm 0.31 \mathrm{~mm}$ and an absolute intra-observer variability average difference of $0.38 \pm 0.17 \mathrm{~mm}$ for cochlear length measurements, on CTscans. Moreover, it is also worthy to notice the resolution of CT-images with a voxel size of $0.625 \times 0.39 \times 0.39 \mathrm{~mm}^{3}$ : errors inferior to such a resolution may thus not be preventable. All this considered together, our mismatches seem to be even inferior to the interindividual anatomical variation itself and may therefore be considered acceptable.

Once the geometry of $3 \mathrm{D}$-printed $\mathrm{TB}$ has been validated, the model can be printed infinitely. Other models could thus be considered: adult or child TB, with normal or unusual anatomy and even with pathologic scenarios. Furthermore, similar TB models could be manufactured based on clinical CT-scans for preoperative simulation of specific challenging cases, potentially reducing medical errors and surgeon stress. But, to do so, the time-consuming manual segmentation step should be overtaken in the future by developing automatization for structure identification, based on atlas (54).

Perspectives of this first study developing objective validation methodology of artificial surgical simulator are numerous. Indeed, the methodology developed here and inspired from the field of engineer sciences could be applied to the other surgical specialties. Moreover, while the present study focused on the geometric characteristics of the artificial surgical simulator, it would be of crucial interest, in the future, to assess the material characteristics, including its response to machining, especially in terms of drilling resistance compared to human bone.

Finally, such simulators, once validated, should be considered, like in aeronautics, for use in standardized testing and compulsory certification (55) before real invivo surgery. Some protocols now stand, like in robotic surgery (56,57), or for learning curves (58-60) by comparing simulator users versus non-users over time.

\section{Conclusions}

As a conclusion, our TB model is reliable regarding anatomy and thus could be considered into ENT surgical education programs. The methodology used in this study is objective, drawn from engineer sciences, making it the first of its kind. The validity of the manufacturing process has been verified in the same time, and could therefore be extended to other specialties, emphasizing the importance of cross-disciplinary collaborations concerning new 
technologies.

\section{Acknowledgments}

We would like to thank Doctor E. Schmitt, NeuroRadiologist in the Regional Hospital-University Center of Nancy, and his team, for his precious help to precise anatomical regions of the temporal bone in CTscans, Doctor M. Roch, E.N.T in the Regional Hospital Center of Colmar, for having been part of the initial project and Sayens Satt for funding part of Marta Szczetynska's study grant.

Funding: None.

\section{Footnote}

Conflicts of Interest: The authors have no conflicts of interest to declare.

Etbical Statement: The authors are accountable for all aspects of the work in ensuring that questions related to the accuracy or integrity of any part of the work are appropriately investigated and resolved.

Open Access Statement: This is an Open Access article distributed in accordance with the Creative Commons Attribution-NonCommercial-NoDerivs 4.0 International License (CC BY-NC-ND 4.0), which permits the noncommercial replication and distribution of the article with the strict proviso that no changes or edits are made and the original work is properly cited (including links to both the formal publication through the relevant DOI and the license). See: https://creativecommons.org/licenses/by-nc-nd/4.0/.

\section{References}

1. Granry JC, Moll MC. Rapport de mission: état de l'art (national et international) en matière de pratiques de simulation dans le domaine de la santé, dans le cadre du développement professionnel continu et de la prévention des risques HAS 2012. Paris, 2012. Available online: https://www.has-sante.fr/portail/upload/docs/application/ pdf/2013-01/guide_bonnes_pratiques_simulation_sante_ format2clics.pdf

2. Levraut J, Fournier JP. Jamais la première fois sur le patient ! Available online: http://link.springer. com/10.1007/s13341-012-0259-9

3. Kamrava B, Roehm PC. Systematic review of ossicular chain anatomy: strategic planning for development of novel middle ear prostheses. Otolaryngol Head Neck Surg 2017;157:190-200.

4. Hohman MH, Bhama PK, Hadlock TA. Epidemiology of iatrogenic facial nerve injury: a decade of experience. Laryngoscope 2014;124:260-5.

5. Alzhrani F, Lenarz T, Teschner M. Facial palsy following cochlear implantation. Eur Arch Otorhinolaryngol 2016;273:4199-207.

6. Migirov L, Eyal A, Kronenberg J. Intracranial complications following mastoidectomy. Pediatr Neurosurg 2004;40:226-9.

7. Senechaut JP, Hazan A, Henrion P, et al. Severe iatrogenic complications of surgery of the middle ear and their medicolegal aspects. Ann Otolaryngol Chir Cervicofac 1988;105:377-82.

8. Mancini F, Taibah AK, Falcioni M. Complications and their management in tympanomastoid surgery. Otolaryngol Clin North Am 1999;32:567-83.

9. McManus LJ, Stringer MD, Dawes PJD. Iatrogenic injury of the chorda tympani: a systematic review. J Laryngol Otol 2012;126:8-14.

10. Scott A, De R, Sadek SA, et al. Temporal bone dissection: a possible route for prion transmission? J Laryngol Otol 2001;115:374-5.

11. Demiryürek D, Bayramoğlu A, Ustaçelebi Ş. Infective agents in fixed human cadavers: A brief review and suggested guidelines. Anat Rec 2002;269:194-7.

12. Arloing $S$, Chauveau A, Fleming G. The comparative anatomy of the domesticated animals. New York: D. Appleton and Company, 1873. Available online: http:// www.biodiversitylibrary.org/bibliography/39284

13. Treuting PM, Dintzis SM, Montine KS. Comparative anatomy and histology: a mouse, rat, and human Atlas. Second edition. London San Diego, Calif: Academic Press, 2018: 552.

14. Varoquier M, Hoffmann CP, Perrenot C, et al. Construct, face, and content validation on Voxel-Man ${ }^{\circledR}$ simulator for otologic surgical training. Int J Otolaryngol 2017;2017:2707690.

15. Kelly DC, Margules AC, Kundavaram CR, et al. Face, content, and construct validation of the da Vinci Skills simulator. Urology 2012;79:1068-72.

16. Lin $\mathrm{Y}$, Wang $\mathrm{X}, \mathrm{Wu} \mathrm{F}$, et al. Development and validation of a surgical training simulator with haptic feedback for learning bone-sawing skill. J Biomed Inform 2014;48:122-9.

17. Fang TY, Wang PC, Liu CH et al. Evaluation of a haptics- 
based virtual reality temporal bone simulator for anatomy and surgery training. Comput Methods Programs Biomed 2014;113:674-81.

18. Bakhos D, Velut S, Robier A, et al. Three-dimensional modeling of the temporal bone for surgical training. Otol Neurotol 2010;31:328-34.

19. Rose AS, Kimbell JS, Webster CE, et al. Multi-material 3D models for temporal bone surgical simulation. Ann Otol Rhinol Laryngol 2015;124:528-36.

20. Suzuki R, Taniguchi N, Uchida F, et al. Transparent model of temporal bone and vestibulocochlear organ made by $3 \mathrm{D}$ printing. Anat Sci Int 2018;93:154-9.

21. Jin SJ, Jeong ID, Kim JH, et al. Accuracy (trueness and precision) of dental models fabricated using additive manufacturing methods. Int J Comput Dent 2018;21:107-13.

22. Rebong RE, Stewart KT, Utreja A, et al. Accuracy of three-dimensional dental resin models created by fused deposition modeling, stereolithography, and polyjet prototype technologies: A comparative study. Angle Orthod 2018;88:363-9.

23. Tappa K, Jammalamadaka U. Novel biomaterials used in medical 3D printing techniques. J Funct Biomater 2018. doi: 10.3390/jfb9010017.

24. Dietrich CA, Ender A, Baumgartner S, et al. A validation study of reconstructed rapid prototyping models produced by two technologies. Angle Orthod 2017;87:782-7.

25. Okada DM, de Sousa AMA, Huertas R de A, et al. Surgical simulator for temporal bone dissection training. Braz J Otorhinolaryngol 2010;76:575-8.

26. Capello E, Semeraro Q. The harmonic fitting method for the assessment of the substitute geometry estimate error. Part I: 2D and 3D theory. Int J Mach Tools Manuf 2001;41:1071-102.

27. Manmadhachary A, Ravi Kumar Y, Krishnanand L. Improve the accuracy, surface smoothing and material adaption in STL file for RP medical models. J Manuf Process 2016;21:46-55.

28. Béchet E, Cuilliere JC, Trochu F. Generation of a finite element MESH from stereolithography (STL) files. Comput Aided Des 2002;34:1-17.

29. Ahuja AT, Yuen HY, Wong KT, et al. Computed tomography imaging of the temporal bone-normal anatomy. Clinical Radiology 2003;58:681-6.

30. Blender. Available online: https://www.blender.org/

31. Slicer. Available online: https://www.slicer.org/

32. VTK. Available online: www.vtk.org

33. Landrigan CP, Rothschild JM, Cronin JW, et al. Effect of reducing interns' work hours on serious medical errors in intensive care units. N Engl J Med 2004;351:1838-48.

34. Fonseca AL, Reddy V, Longo WE, et al. Operative confidence of graduating surgery residents: a training challenge in a changing environment. Am J Surg 2014;207:797-805.

35. Ahmed N, Devitt KS, Keshet I, et al. A systematic review of the effects of resident duty hour restrictions in surgery: impact on resident wellness, training, and patient outcomes. Ann Surg 2014;259:1041-53.

36. Correia JC, Steyl JL, De Villiers HC. Assessing the survival of Mycobacterium tuberculosis in unembalmed and embalmed human remains. Clin Anat 2014;27:304-7.

37. Whitehead MC, Savoia MC. Evaluation of methods to reduce formaldehyde levels of cadavers in the dissection laboratory. Clin Anat 2008;21:75-81.

38. Naz S, Nazir G, Iram S, et al. Perceptions of cadaveric dissection in anatomy teaching. J Ayub Med Coll Abbottabad 2011;23:145-8.

39. Arora A, Swords C, Khemani S, et al. Virtual reality casespecific rehearsal in temporal bone surgery: a preliminary evaluation. Int J Surg 2014;12:141-5.

40. Suzuki M, Ogawa Y, Kawano A, et al. Rapid prototyping of temporal bone for surgical training and medical education. Acta Oto-Laryngologica 2004;124:400-2.

41. Wang H, Northrop C, Burgess B, et al. Threedimensional virtual model of the human temporal bone: a stand-alone, downloadable teaching tool. Otol Neurotol 2006;27:452-7.

42. Suzuki M, Hagiwara A, Ogawa Y, et al. Rapid-prototyped temporal bone and inner-ear models replicated by adjusting computed tomography thresholds. J Laryngol Otol 2007;121:1025-8.

43. Mick PT, Arnoldner C, Mainprize JG, et al. Face validity study of an artificial temporal bone for simulation surgery. Otol Neurotol 2013;34:1305-10.

44. Hochman JB, Kraut J, Kazmerik K, et al. Generation of a $3 \mathrm{D}$ printed temporal bone model with internal fidelity and validation of the mechanical construct. Otolaryngol Head Neck Surg 2014;150:448-54.

45. Ahmed K, Keeling AN, Fakhry M, et al. Role of virtual reality simulation in teaching and assessing technical skills in endovascular intervention. J Vasc Interv Radiol 2010;21:55-66.

46. Okrainec A, Farcas M, Henao O, et al. Development of a virtual reality haptic veress needle insertion Simulator for surgical skills training. Stud Health Technol Inform 2009;142:233-8. 
47. Oropallo W, Piegl LA. Ten challenges in 3D printing. Eng Comput 2016;32:135-48.

48. Yao AWL. Applications of 3D scanning and reverse engineering techniques for quality control of quick response products. Int J Adv Manuf Technol 2005;26:1284-8.

49. Würfel W, Lanfermann H, Lenarz T, et al. Cochlear length determination using Cone Beam Computed Tomography in a clinical setting. Hear Res 2014;316:65-72.

50. Singh D, Hsu CCT, Kwan GNC, et al. High resolution CT study of the chorda tympani nerve and normal anatomical variation. Jpn J Radiol 2015;33:279-86.

51. Iyaniwura JE, Elfarnawany M, Riyahi-Alam S, et al. Intra- and Interobserver Variability of Cochlear Length Measurements in Clinical CT. Otol Neurotol 2017;38:828-32.

52. Quam RM, Coleman MN, Martínez I. Evolution of the auditory ossicles in extant hominids: metric variation in African apes and humans. J Anat 2014;225:167-96.

53. Frangi AF, Rueckert D, Schnabel JA, et al. Automatic construction of multiple-object three-dimensional statistical shape models: application to cardiac modeling. IEEE Trans Med Imaging 2002;21:1151-66.

54. Powell KA, Liang T, Hittle B, et al. Atlas-Based Segmentation of Temporal Bone Anatomy. Int J Comput

Cite this article as: Chauvelot J, Laurent C, Le Coz G, Jehl JP, Tran N, Szczetynska M, Moufki A, Bonnet AS, Parietti-Winkler C. Morphological validation of a novel bi-material 3D-printed model of temporal bone for middle ear surgery education. Ann Transl Med 2020;8(6):304. doi: 10.21037/atm.2020.03.14
Assist Radiol Surg 2017;12:1937-44.

55. Abou-Elhamd KEA, Al-Sultan AI, Rashad UM. Simulation in ENT medical education. J Laryngol Otol 2010;124:237-41.

56. Hubert J, Perrenot C, Trand N, et al. Simulation chirurgicale ( $\mathrm{dv}$-trainer $\AA)$ et formation chirurgicale robotique. E-memoire de L'Academie Nationale de Chirurgie 2012;11:80-4.

57. Rapoport LM, Bezrukov EA, Tsarichenko DG, et al. Methods for training of robot-assisted radical prostatectomy. Available online: http://www.mediasphera. ru/issues/khirurgiya-zhurnal-im-n-i-pirogova/2019/1/ downloads/ru/1002312072019011089

58. Gromski MA, Cohen J, Saito K, et al. Learning colorectal endoscopic submucosal dissection: a prospective learning curve study using a novel ex vivo simulator. Surg Endosc 2017;31:4231-7.

59. Colaco HB, Hughes K, Pearse E, et al. Construct validity, assessment of the learning curve, and experience of using a low-cost arthroscopic surgical simulator. J Surg Educ 2017;74:47-54.

60. Podolsky DJ, Fisher DM, Wong Riff KW, et al. Assessing technical performance and determining the learning curve in cleft palate surgery using a high-fidelity cleft palate simulator. Plast Reconstr Surg 2018;141:1485-500. 